\title{
Fermentation Studies of Rustmicin Production by a Micromonospora sp.
}

\author{
J. M. Sigmund* and C. F. HiRSCH \\ Department of Natural Products Drug Discovery, Merck Research Laboratories, \\ R80Y-330 P.O. Box 2000, Rahway, New Jersey 07065, U.S.A.
}

(Received for publication March 30, 1998)

\begin{abstract}
The antifungal antibiotic rustmicin was detected in the fermentation broth of the actinomycete MA 7094 as a specific inhibitor of sphingolipid biosynthesis in Candida albicans and as a potent fungicidal agent against Cryptococcus neoformans. Taxonomic characterization by both classical means and PCR fingerprinting supported the assignment of the producing culture to the genus Micromonospora. Fermentation medium optimization studies showed that the concentration of tomato paste in the medium was critical to increased production of rustmicin by MA 7094. The stimulatory effect of tomato paste in the medium on rustmicin production appeared to be related to the maintenance of $\mathrm{pH}$ at or below a value of 6.0. Addition of the antifoam agent P-2000 to the fermentation was found to dramatically reduce the rustmicin titer, while substitution of another antifoam agent, UCON-LB625, resulted in a $100 \%$ increase in the amount of rustmicin detected. After fermentation optimization studies and the generation of a non-sporulating mutant of MA 7094, the rustmicin titer was increased from an initial titer of $10 \mathrm{mg} /$ liter to $145 \mathrm{mg} /$ liter.
\end{abstract}

During the course of screening for novel antimicrobial compounds from natural sources, an antifungal compound was discovered in the fermentation broth of Micromonospora strain MA 7094. The compound was isolated and identified as the 14-membered macrolide antifungal antibiotic rustmicin. Rustmicin was originally described by TAKATSU et al. as an inhibitor of the wheat stem rust fungus Puccinia graminis ${ }^{1}$ and was detected in a fermentation broth of Micromonospora chalcea 980$\mathrm{MC}_{1}{ }^{2)}$. Shortly thereafter, galbonolide $\mathrm{A}$ was reported in the literature as a new antifungal macrolide produced by Streptomyces galbus $^{3}$. The antifungal activity of galbonolide $\mathrm{A}$ was demonstrated against a battery of phytopathogenic fungi and was most effective against Botrytis cinerea. Galbonolide A was later found to be identical in structure to rustmicin.

Studies described by ACHENBACH et al. ${ }^{4)}$ indicated that the antifungal action of rustmicin was not due to cell membrane destabilization, the inhibition of chitin biosynthesis or interference with DNA or RNA biosynthesis. The mode of action of rustmicin was unknown. Recently, it has been determined that rustmicin specifically inhibits sphingolipid biosynthesis in Candida albicans and is a potent fungicidal agent of the human pathogen Cryptococcus neoformans ${ }^{5)}$. These novel findings on the mode of action and bioactivity of rustmicin initiated the following study on the production of this antibiotic by MA 7094. This paper reports the taxonomy of the producing culture, fermentation medium optimization and the effect of antifoaming agents on rustmicin production.

\section{Materials and Methods}

\section{Microorganisms}

The rustmicin-producing culture was isolated from a soil sample collected in New Hampshire, USA and deposited in the Merck Culture Collection as a Micromonospora sp. with the accession number MA 7094. A high-titer producing strain of MA 7094 was isolated following UV mutagenesis treatment as described below and was deposited in the Merck Culture Collection with the accession number MA 7186. The following type strains of Micromonospora used for comparative taxonomic studies were obtained from the ATCC: Micromonospora carbonacea 27114, M. carbonacea ssp. aurantiaca 27115 , M. echinospora ssp. echinospora 15837 , M. coerulea 27008, M. brunnea 27334, M. purpurea 15835 , M. purpureochromogenes 27007, M. olivasterospora 21819, M. citrea 35571, M. aurantiaca 27029, M. chalcea 
12452, M. halophytica ssp. halophytica 27596, M. halophytica ssp. nigra 33088, M. inositola 21773, $M$. rhodorangea 27932 , and $M$. rosaria 29337 . The following cultures were obtained from the NRRL: M. echinospora ssp. ferruginea 2995 and M. echinospora ssp. pallida 2996. All cultures were preserved as vegetative mycelia in $10 \%(\mathrm{v} / \mathrm{v})$ glycerol and stored at $-80^{\circ} \mathrm{C}$.

\section{Taxonomic Studies}

Observations of growth and general cultural characteristics of MA7094, as well as the determination of carbon utilization pattern, were done according to the procedures described by SHIRLING and GotTleib ${ }^{6)}$. All polymerase chain reaction (PCR) banding patterns were generated and visualized using the procedures described by HiRsCH and SigmUND for low resolution fingerprinting ${ }^{7)}$. Primers used for low resolution PCR fingerprinting, T5A and T3B, were described by WELSH and MCCLELLAND $^{8,9)}$ and have extensive homology to tRNA genes. High resolution PCR fingerprinting was done using the Enterobacterial Repetitive Intergenic Consensus (ERIC) primer set, ERIC1R and ERIC2, described by Versalovic et al. ${ }^{10)}$ and Louws et al. ${ }^{11)}$. All primers were purchased from GENSET (La Jolla, CA, USA). Template DNA used in PCR was extracted from cells by incubating them in water at $95^{\circ} \mathrm{C}$ for 30 minutes as described previously ${ }^{7)}$. In some instances, template DNA was diluted in the range of $1: 4$ to $1: 20$ in order to optimize the high resolution banding patterns. Dilution can be used to decrease the concentration of inhibitors of the PCR reaction that may be present in the crude DNA extract. PCR fingerprint patterns were analyzed using the GelCompar software package from Applied Maths (Risquons-Toutstraat 38, B-8511 Kortrijk, Belgium).

\section{Fermentation}

A seed culture was produced by inoculating $50 \mathrm{ml}$ of aqueous nutrient medium in a $250 \mathrm{ml}$ triple baffled Erlenmeyer flask with $2 \mathrm{ml}$ of thawed frozen vegetative mycelia. The nutrient medium contained glucose $10 \mathrm{~g}$, soluble starch $20 \mathrm{~g}$, yeast extract $5 \mathrm{~g}, \mathrm{~N}-\mathrm{Z}$ amine A $5 \mathrm{~g}$, beef extract $3 \mathrm{~g}$, bacto-peptone $5 \mathrm{~g}$, and $\mathrm{CaCO}_{3} 1 \mathrm{~g}$ in 1 liter of deionized water. The $\mathrm{pH}$ of the medium was adjusted to 7.0 with $\mathrm{NaOH}$ prior to $\mathrm{CaCO}_{3}$ addition. After inoculation, the seed culture was incubated at $28^{\circ} \mathrm{C}$ and shaken at $220 \mathrm{rpm}$ for 72 hours. A volume of $2 \mathrm{ml}$ of the seed culture was aseptically transferred to $44 \mathrm{ml}$ of production medium in a $250 \mathrm{ml}$ non-baffled Erlenmeyer flask. The original production medium, KHC, contained dextrin $20 \mathrm{~g}$, beta-cyclodextrin $10 \mathrm{~g}$, primary yeast $10 \mathrm{~g}$, tomato paste (Hunt's) $20 \mathrm{~g}$, and $\mathrm{CoCl}_{2} \cdot 6 \mathrm{H}_{2} \mathrm{O}$ $0.005 \mathrm{~g}$ in 1 liter of deionized water, adjusted to $\mathrm{pH} 7.2$ with $\mathrm{NaOH}$. Optimized production medium, OPT14, contained dextrin $25 \mathrm{~g}$, beta-cyclodextrin $10 \mathrm{~g}$, primary yeast $14 \mathrm{~g}$, tomato paste $4 \mathrm{~g}, \mathrm{CoCl}_{2} \cdot 6 \mathrm{H}_{2} \mathrm{O} 0.005 \mathrm{~g}$, and UCON-LB $6254 \mathrm{ml}$ in 1 liter of deionized water, adjusted to $\mathrm{pH} 7.2$ with $\mathrm{NaOH}$. Cultures in production media were incubated at $28^{\circ} \mathrm{C}$ and shaken at $220 \mathrm{rpm}$ for 5 or 6 days. UCON-LB625 (UCON) was purchased from Union Carbide Corporation, USA.

\section{Analysis of Fermentation Samples}

The amount of ammonia present in fermentation broth was determined by HPLC analysis of clarified broth using a HPLC-CS4 column (Dionex Corp., USA). The column effluent was mixed with $o$-phthalaldehyde (Fluoraldehyde, Pierce Chemical Co., USA) and compounds possessing primary amino groups were derivatized and measured using a fluorescence detector. The concentration of ammonia was calculated from a standard curve generated using $\mathrm{NH}_{4} \mathrm{Cl}$.

Rustmicin production was determined using reverse phase HPLC ${ }^{12)}$. Fermentation broth samples were prepared for assay by addition of an equal volume of methanol, agitation for 1 hour and clarification by filtration or centrifugation. Reverse phase HPLC was performed on a Phenomenex Primesphere C8 column, $4.6 \times 250 \mathrm{~mm}$ using a mobile phase of methanol $/ 25 \mathrm{~mm}$ $\mathrm{NH}_{4} \mathrm{OAc}(75 / 25)$, a flow rate of $1.0 \mathrm{ml} / \mathrm{minute}$ and a column temperature of $40^{\circ} \mathrm{C}$. UV detection was performed at $235 \mathrm{~nm}$. Rustmicin eluted at 13.0 minutes.

\section{UV Mutagenesis}

Spores of MA 7094 were suspended in sterile water at a concentration of $1.9 \times 10^{7}$ colony forming units (cfu). A volume of $10 \mathrm{ml}$ of spore suspension was placed in a sterile $100 \mathrm{~mm}$ dia. glass petri dish on a rotating table and irradiated for 20 seconds with UV light $(254 \mathrm{~nm}, 5$ mjoules/second). This treatment reduced the cfu of the spore suspension to about $1 \%$ of the initial count. Following irradiation, samples of the spore suspension were plated onto BAM agar medium (glucose $10 \mathrm{~g}, \mathrm{~N}-\mathrm{Z}$ Amine A $2 \mathrm{~g}$, beef extract $1 \mathrm{~g}$, yeast extract $1 \mathrm{~g}$ and agar $15 \mathrm{~g}$ in 1 liter distilled water, $\mathrm{pH}$ 7.3) and incubated at $28^{\circ} \mathrm{C}$ in the dark. After incubation for $7 \sim 10$ days, the colonies on the plates were examined and those which exhibited bright orange pigmentation and lacked the black to dark gray colored spore masses were picked. The cultures were examined microscopically for the 
presence of spores, and those which exhibited no or very reduced sporulation were selected for fermentaion.

\section{Results}

Morphological and Cultural Characteristics

$$
\text { of MA } 7094
$$

Strain MA 7094 stains Gram-positive and forms well-developed, branched vegetative mycelia. Aerial mycelia were not observed. Microscopic examination of MA 7094 revealed spores that were non-motile and spherical to oval in shape. Spores of MA 7094 were formed singly on substrate mycelium and were located terminally on short hyphal branches.

The cultural characteristics of MA 7094 were determined by macroscopic observation of growth on various differential media routinely employed in taxonomic studies of actinomycetes ${ }^{6)}$. Colonies of MA 7094 on agar media often had raised centers with a filamentous margin, and vegetative mycelia were light orange to brown orange in color. Mature colonies were brown or black in appearance due to the formation of spores in large, black mucoid masses over the colony surface. No diagnostic mycelial or soluble pigments were observed under the growth conditions employed.

The results of the morphological and cultural studies of MA 7094 were consistent with those described in the literature for the genus Micromonospora ${ }^{13,14)}$.

\section{PCR Fingerprinting of MA 7094}

PCR fingerprinting of MA 7094 was done in an attempt to support the identification of the culture as a Micromonospora and to determine its relationship to various type strains of the genus. Using GelCompar, the

Fig. 1. Comparison of PCR fingerprint of MA 7094 and $M$. purpureochromogenes ATCC $27007, M$. inositola ATCC 21773, M. citrea ATCC 35571, M. brunnea ATCC 27334, and M. halophytica ssp. halophytica ATCC 27596.

(A) Low resolution PCR fingerprinting. (B) High resolution PCR fingerprinting.

A



base pairs

MA 7094

ATCC 27596

ATCC 27334

ATCC 35571

ATCC 27007

ATCC 21773

B

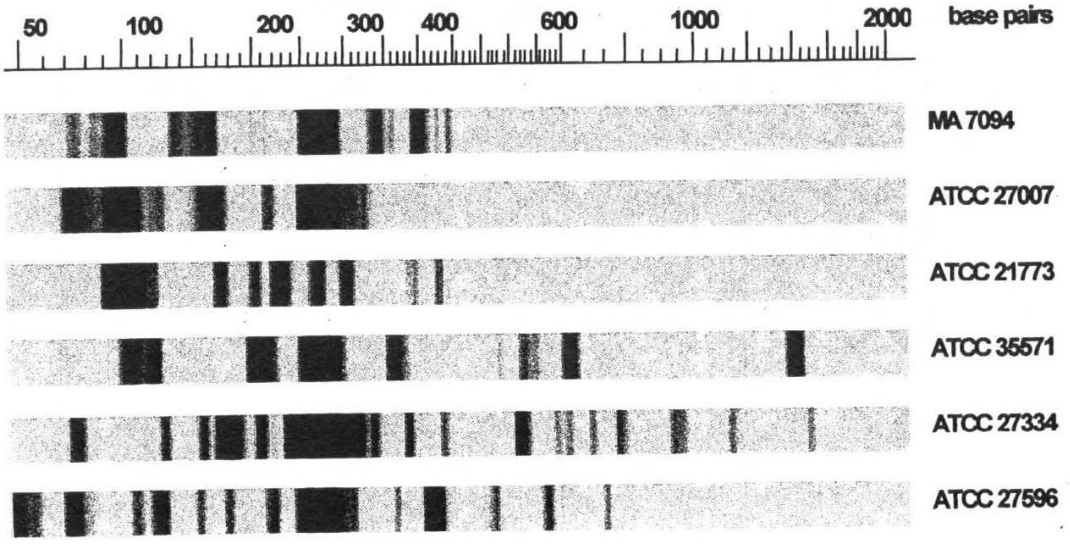


low resolution fingerprint pattern of MA 7094 was compared to those of over 550 different type strains of Gram-negative and Gram-positive bacteria, including over 30 different genera of actinomycetes and 18 type strains of Micromonospora. The low resolution PCR fingerprint most closely matching that of MA 7094 was that of Micromonospora purpureochromogenes (96.4\% similarity). The next four closest matches (92.2 85.4\% similarity) also were species of Micromonospora (Fig. 1A). The fingerprint banding patterns among the different strains were very similar in that three distinct bands located between 95 bp and 290 bp were present for each culture.

The close similarity of the low resolution PCR fingerprint of MA 7094 to five different species of Micromonospora suggested that high resolution PCR fingerprinting might be used to identify MA 7094 to species level. High resolution PCR fingerprints were generated for MA 7094 and each culture listed in Fig. 1A. A comparison of the high resolution banding patterns

Fig. 2. Effect of medium components on the production of rustmicin by MA 7094 .

(A) Effect of dextrin and primary yeast concentration on rustmicin production.

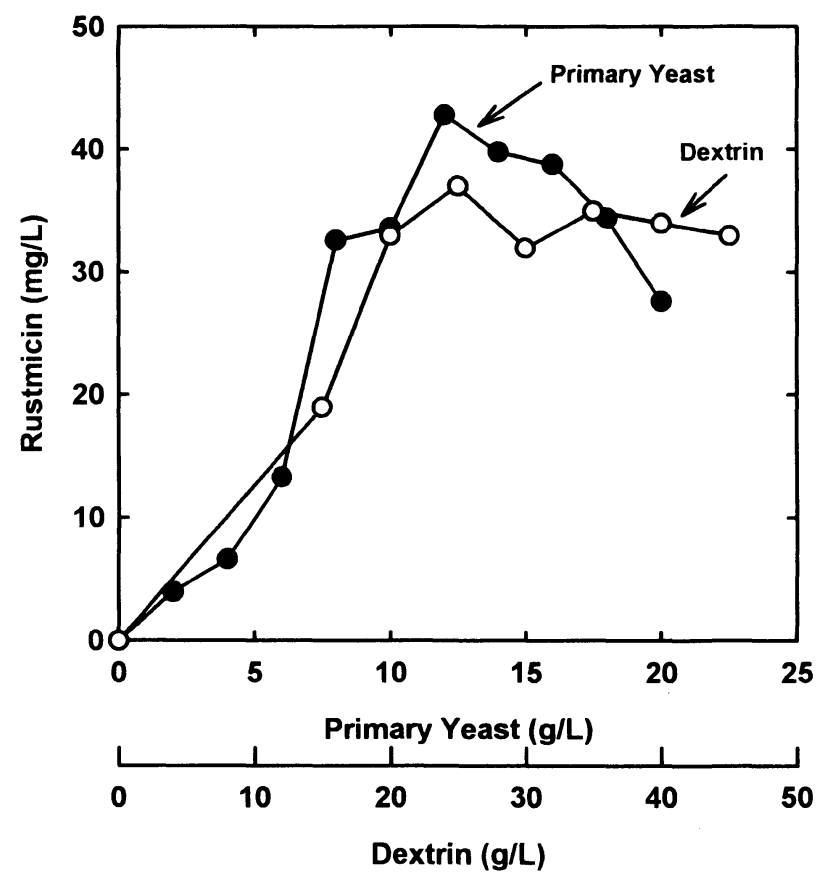

(B) Effect of tomato paste concentration on $\mathrm{pH}$ and rustmicin production.

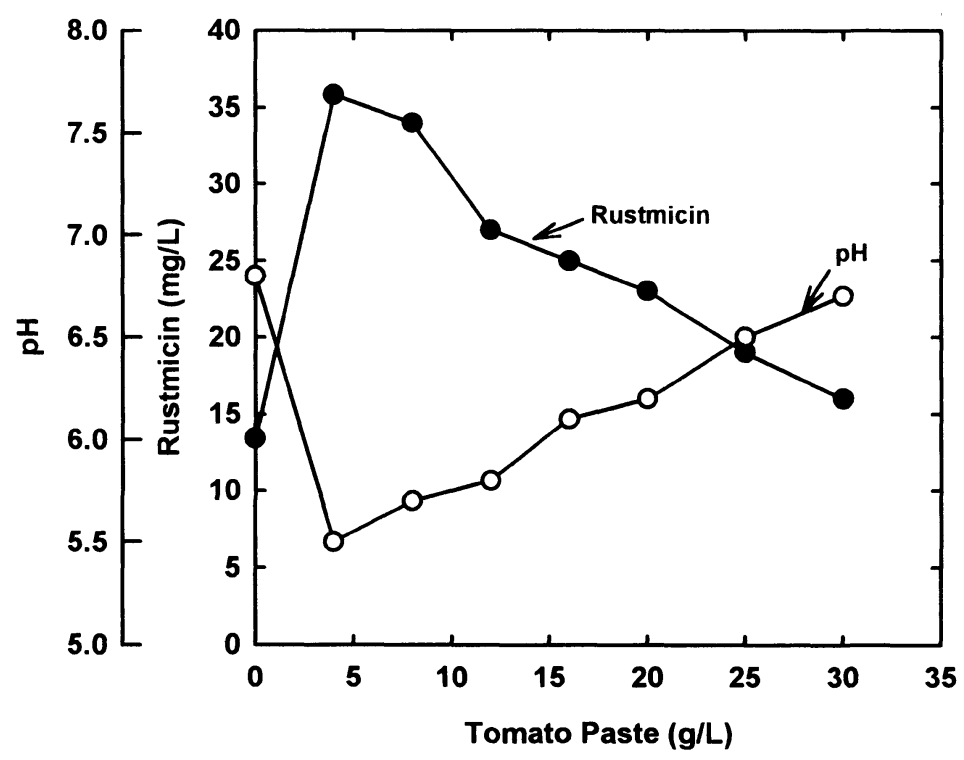


shown in Fig. 1B revealed a very different pattern for each culture in terms of the number and position of bands.

\section{Medium Optimization Studies}

The effect of dextrin and primary yeast concentration on the production of rustmicin by MA 7094 was studied by varying the amounts of dextrin or primary yeast in KHC production medium while maintaining the concentrations of the other medium components constant. After 5 days of incubation, $\mathrm{pH}$ and rustmicin levels were determined and the results are shown in Fig. 2A. Without the addition of dextrin or primary yeast to the production medium, no rustmicin was detected. Rustmicin production increased with increasing amounts of dextrin and primary yeast up to a level of approximately $25 \mathrm{~g} /$ liter and $12 \mathrm{~g} /$ liter respectively. At a dextrin concentration of $25 \mathrm{~g} /$ liter, the titer of rustmicin approached $36 \mathrm{mg} /$ liter and then remained constant in media containing dextrin levels up to $45 \mathrm{~g} /$ liter. A maximum rustmicin titer of $43 \mathrm{mg} /$ liter was obtained at a primary yeast concentration of $12 \mathrm{~g} /$ liter. Rustmicin titer decreased in media containing levels of primary yeast above $16 \mathrm{~g} /$ liter. The $\mathrm{pH}$ of $\mathrm{KHC}$ production medium after 5 days of incubation was not significantly affected by varying the concentration of dextrin or primary yeast in the medium (data not shown).

A similar experiment was done to determine the effect of tomato paste on the production of rustmicin by MA 7094. KHC production media containing increasing amounts of tomato paste were inoculated with MA 7094.
After five days of incubation, $\mathrm{pH}$ and rustmicin levels were determined. As shown in Fig. 2B, increasing the concentration of tomato paste in the production medium from $4 \mathrm{~g} /$ liter to $30 \mathrm{~g} /$ liter had an inverse effect on rustmicin production and produced a concomitant increase in medium $\mathrm{pH}$. A maximum rustmicin titer of $36 \mathrm{mg} /$ liter was observed in medium containing $4 \mathrm{~g} /$ liter tomato paste, with a corresponding $\mathrm{pH}$ of 5.4. Tomato paste appeared not to be essential for the production of rustmicin by MA 7094, since rustmicin was detected in medium containing no tomato paste. Low levels of tomato paste, however, did appear to stimulate rustmicin production by MA 7094 and may serve to lower the $\mathrm{pH}$ of the medium to a level where rustmicin is most stable. Attempts to buffer production medium at or below $\mathrm{pH}$ 6.0 with phosphate, $\mathrm{CaCO}_{3}$, PIPES or MES failed to increase the amount of rustmicin detected when compared to the amount detected in KHC medium without added buffers (data not shown).

The time course of rustmicin production by MA 7094 incubated in medium containing the optimized concentrations of dextrin, primary yeast, and tomato paste (OPT 14) is shown in Fig. 3. Rustmicin production began at about 40 hours and reached a maximum titer of $38 \mathrm{mg} /$ liter at 140 hours of incubation. The $\mathrm{pH}$ of the medium at the start of rustmicin production was approximately 5.7 , and it remained relatively constant throughout rustmicin production until 120 hours of incubation. After 120 hours, a dramatic increase in $\mathrm{pH}$ from 5.7 to 7.6 at 160 hours of incubation was observed, accompanied by a decline in the rustmicin titer. The

Fig. 3. Time course of rustmicin production by MA 7094 in OPT 14 medium.

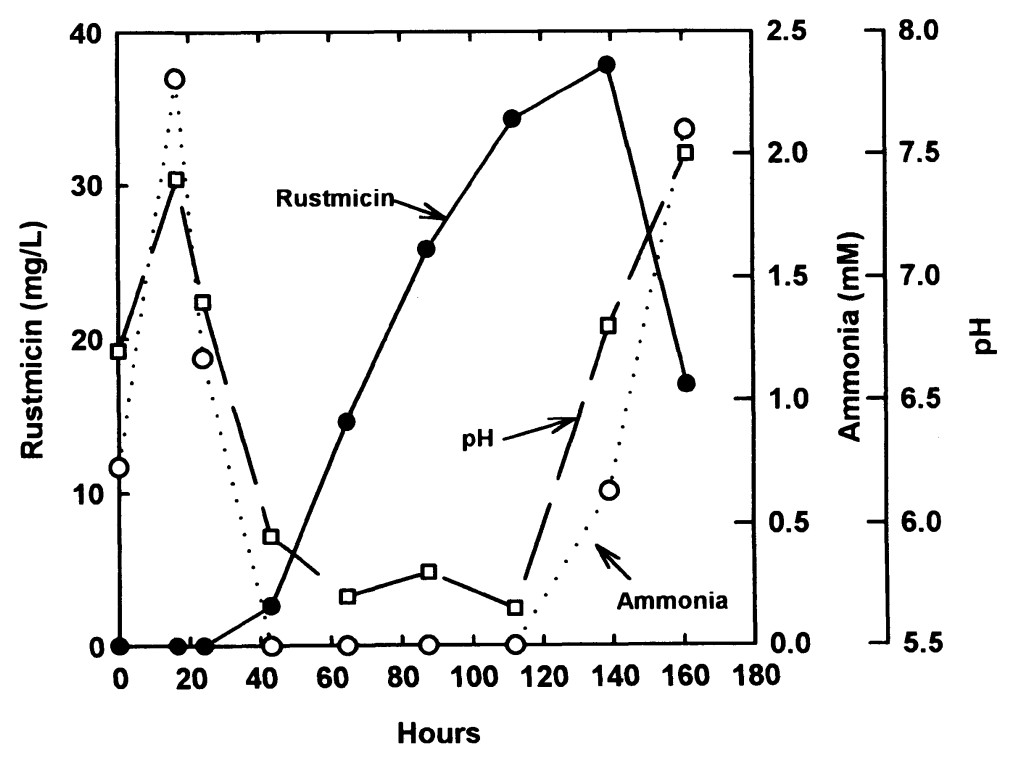


Table 1. Effect of antifoams on $\mathrm{pH}$ and production of rustmicin by MA 7094.

\begin{tabular}{llccc}
\hline Antifoam & $\begin{array}{c}\text { Antifoam } \\
\% \mathrm{v} / \mathrm{v}\end{array}$ & $\mathrm{pH}$ & $\begin{array}{c}\text { Rustmicin } \\
\% \text { decrease }\end{array}$ & $\begin{array}{c}\text { Rustmicin } \\
\% \text { increase }\end{array}$ \\
\hline No addition & & 5.7 & & \\
P-2000 & 0.05 & 5.9 & 49 & \\
P-2000 & 0.1 & 6.5 & 64 & \\
P-2000 & 0.15 & 7.2 & 87 & \\
P-2000 & 0.2 & 7.7 & 95 & \\
Hodag FD-62 & 0.1 & 5.9 & & \\
SAG-471 & 0.1 & 6.3 & $<10$ & \\
UCON-LB625 & 0.1 & 5.8 & & \\
MAZU-DF201 & 0.1 & 6.4 & 15 & 13 \\
SIGMA-A & 0.1 & 6.3 & $<10$ & $<10$ \\
SIGMA-B & 0.1 & 6.0 & & \\
SIGMA-C & 0.1 & 6.0 & & \\
SIGMA-204 & 0.1 & 6.4 & & \\
\hline
\end{tabular}

$\mathrm{pH}$ and rustmicin concentration determined after 5 days of incubation in OPT 14 medium.

increase in $\mathrm{pH}$ after 120 hours was observed to correlate with an increase in the concentration of ammonia detected in the fermentation, which reached a concentration of $2.2 \mathrm{~mm}$ at 160 hours of incubation.

\section{Effect of Antifoam Agents on \\ Rustmicin Production}

In preparation for scale-up of the fermentation, the effect of various antifoam agents on rustmicin production by MA 7094 incubated in OPT 14 medium for 5 days was studied. Table 1 shows that the addition of $0.05 \%(\mathrm{v} / \mathrm{v}) \mathrm{P}-2000$ to the production medium caused a $49 \%$ decrease in the amount of rustmicin detected. The inhibitory effect of $0.05 \% \mathrm{P}-2000$ on rustmicin production did not appear to be related to an increase in $\mathrm{pH}$. Increasing the concentration of P-2000 above $0.05 \%$ resulted in further decreases in rustmicin production and a concomitant rise in $\mathrm{pH}$ of the medium. The addition of the antifoam agents Hodag FD-62, SAG-471, MAZU-DF201, SIGMA-A, SIGMA-C and SIGMA-204 did not cause a significant change in the amount of rustmicin detected in the fermentation, but when SIGMA-B or UCON-LB625 was added to the medium, an increase in rustmicin titer of $30 \%$ and $40 \%$, respectively, was observed.

The stimulation of rustmicin production by UCON was investigated in more detail. An experiment was designed to test the effect of increasing amounts of UCON on rustmicin titer and pH in OPT 14 medium. These experiments were done using MA 7186, a UV generated mutant of MA 7094 discussed below which consistently produced $50 \%$ greater rustmicin titers than its parent using identical growth conditions (data not shown). The results are shown in Table 2. Generally, significant increases in rustmicin titer were observed in media containing $\geq 0.3 \%$ UCON when compared to unsupplemented media after 5 days of incubation. More specifically, in the presence of $\geq 0.4 \%$ UCON at 6 days, rustmicin titer exhibited a two-fold increase from that obtained in medium without UCON. No differences were observed in the $\mathrm{pH}$ of medium with or without UCON supplementation after 5 days of incubation. However, after 6 days of incubation, the $\mathrm{pH}$ of unsupplemented medium was significantly higher than that of media containing $\geq 0.2 \%$ UCON.

\section{UV Mutagenesis of MA 7094}

During the course of the medium optimization studies, it was observed that rustmicin was rarely detected under conditions in which sporulation of the culture had occurred. Based on this observation, it was thought that a non-sporulating mutant of MA 7094 might produce higher rustmicin titers. To test this idea, over $250 \mathrm{UV}$ generated mutants of MA 7094 were screened. One non-sporulating mutant, MA 7186, was found which produced rustmicin titers in OPT 14 medium which were 1.5 to 2-fold higher when compared to those produced by MA 7094. In OPT 14 medium supplemented with $0.4 \%$ UCON, MA 7186 was found to produce a maximum rustimicin titer that was 10 -fold greater (145 
Table 2. Effect of UCON on $\mathrm{pH}$ and production of rustmicin by MA 7186 .

\begin{tabular}{ccccc}
\hline $\begin{array}{c}{[\mathrm{UCON}]} \\
\% \mathrm{v} / \mathrm{v}\end{array}$ & $\begin{array}{c}\mathrm{pH} \\
\text { day } 5\end{array}$ & $\begin{array}{c}\text { Rustmicin } \\
(\mathrm{mg} / \mathrm{liter}) \\
\text { day } 5\end{array}$ & $\begin{array}{c}\mathrm{pH} \\
\text { day } 6\end{array}$ & $\begin{array}{c}\text { Rustmicin } \\
(\mathrm{mg} / \mathrm{liter}) \\
\text { day 6 }\end{array}$ \\
\hline 0 & 5.8 & 42.0 & 6.4 & 47.6 \\
0.1 & 5.8 & 31.9 & 6.1 & 42.7 \\
0.2 & 5.7 & 43.1 & 5.8 & 58.5 \\
0.3 & 5.7 & 71.6 & 5.8 & 69.1 \\
0.4 & 5.7 & 47.1 & 5.8 & 93.8 \\
0.5 & 5.7 & 67.6 & 5.9 & 128 \\
0.6 & 5.8 & 55.1 & 5.9 & 82.4 \\
0.7 & 5.8 & 59.2 & 5.9 & 92.1 \\
0.8 & 5.8 & 74.3 & 5.9 & 105 \\
\hline \multicolumn{5}{c}{ pH and rustmicin concentration determined after 5 days and 6 days } \\
of incubation in OPT 14 medium.
\end{tabular}

$\mathrm{mg} /$ liter) than that originally observed for MA 7094.

\section{Discussion}

Morphological and cultural studies clearly showed that MA 7094 is a member of the genus Micromonospora. However, when physiological characteristics of MA 7094 such as pigment production, carbohydrate utilization and $\mathrm{NaCl}$ tolerance were compared to type species of Micromonospora, it was found that MA 7094 did not closely resemble any of the twelve species of Micromonospora described in the 9th edition of BERGEY's Manual.

In an attempt to differentiate MA 7094 by molecular means, low and high resolution PCR fingerprinting was used. The combination of both types of PCR offers a hierarchical system of culture differentiation ${ }^{7,15}$. Low resolution PCR fingerprinting supported the identification of MA 7094 as a member of the genus Micromonospora since the fingerprints most closely matching that of MA 7094 all belonged to members of this genus. High resolution PCR fingerprinting of MA 7094 and the type strains of Micromonospora which it matched allowed the further differentiation of these cultures. The high resolution fingerprinting showed that each type species of Micromonospora was unique and that each was different from MA 7094.

Earlier reports of rustmicin production indicated that low titers of compound were present in the fermentation broth of $M$. chalcea $980-\mathrm{mc}_{1}{ }^{2)}$ and Streptomyces galbus $^{4}$. Rustmicin titers $\leq 15 \mathrm{mg} /$ liter were detected in the initial fermentation of MA 7094 in KHC production medium. In order to increase the titer of rustmicin produced by MA 7094, over 120 different media were screened. Rustmicin was detected only in those media where carbon was supplied as glucose, maltose, soluble starch or dextrin at titers less than or equal to that detected in KHC medium. Optimization studies of KHC medium showed that rustmicin titers reached a maximum in medium containing approximately $25 \mathrm{~g} /$ liter of dextrin and $14 \mathrm{~g} /$ liter of primary yeast. The concentration of tomato paste in the medium exhibited the most dramatic effect on the fermentation. Reducing the concentration of tomato paste from $20 \mathrm{~g} /$ liter to $4 \mathrm{~g} /$ liter caused a $56 \%$ increase in rustmicin titer with an accompanying reduction in $\mathrm{pH}$ from 6.3 to 5.4. The change in $\mathrm{pH}$ during the fermentation of MA 7094 was observed to correlate closely with the amount of ammonia detected in the medium. Presumably the reduction of tomato paste in the medium directly or indirectly caused a corresponding reduction in the amount of ammonia detected in the fermentation, but the reason for this is not known. The change in ammonia level during the fermentation apparently was not due to carbon limitation since at least $2.5 \mathrm{~mm}$ maltose was detected in the medium at 160 hours of incubation (data not shown).

The dramatic effect of tomato paste on rustmicin production and medium $\mathrm{pH}$ indicated that $\mathrm{pH}$ was an important factor which influenced the amount of rustmicin detected in the fermentation. Studies showed that rustmicin was initially detected in the fermentation of MA 7094 when the $\mathrm{pH}$ of the medium dropped below 6.0 and that the titer declined rapidly when the $\mathrm{pH}$ rose above 6.0. A similar effect of $\mathrm{pH}$ on rustmicin titer was 
observed in the fermentation of $S$. galbus ${ }^{3)}$, and ACHENBACH et al. have shown that pure rustmicin is most stable at $\mathrm{pH} 5.5$ and is extremely labile at $\mathrm{pH}$ levels above $6.0^{4)}$. Therefore, it is likely that the production of rustmicin by MA 7094 in OPT 14 medium may have continued beyond 120 hours of incubation, but was not observed due to its degradation.

The speculation that increased titers of rustmicin may be obtained by $\mathrm{pH}$ maintenance at or below 6.0 for an extended period of time is supported by the observation that the rustmicin titer detected in OPT 14 medium supplemented with $0.4 \%$ or $0.5 \%$ UCON doubled between 5 and 6 days of incubation when the $\mathrm{pH}$ remained below 6.0, while the titer increased only slightly in unsupplemented medium during the same time interval when the $\mathrm{pH}$ increased from 5.8 to 6.4. After 7 days incubation, the $\mathrm{pH}$ of medium containing $0.4 \%$ UCON was 6.15 while that of medium without UCON added was 7.4 (data not shown). The mechanism by which UCON might maintain or contribute to the lowered $\mathrm{pH}$ detected in the fermentation of MA 7186 in OPT 14 medium is not known and requires further study.

\section{Acknowledgements}

We are grateful to GUY HARRIS for providing HPLC methodology, as well as Suzanne Mandala, James Milligan and ROSEMARY THORNTON and for providing bioassay support and unpublished data.

\section{References}

1) Takatsu, T.; H. Nakayama, A. Shimazu, K. Furihata, K. IKeda, K. Furihata, H. Seto \& N. Otake: Rustmicin, a new macrolide antibiotic active against wheat stem rust fungus. J. Antibiotics 38: 1806 1809, 1985

2) Nakayama, H.; T. Takatsu, Y. Abe, A. Shimazu, K. Furihata, K. IKeda, K. Furihata, H. Seto \& N. Otake: Rustmicin, a new macrolide antibiotic active against wheat stem rust fungus. Agric. Biol. Chem. 51: $853 \sim 859$, 1987

3) FAuTh, U. \& H. ZAHner: Galbonolides A and B-Two non-glycosidic antifungal macrolides. J. Antibiotics 39: $1760 \sim 1764,1986$

4) Achenbach, H.; A. Muhlenfeld, U. Fauth \& $\mathrm{H}$. ZAHNER: The Galbonolides. Novel, powerful antifungal macrolides from Streptomyces galbus ssp. eurythermus. In Annals of the New York Academy of Sciences. Antifungal Drugs. Ed., V. St. Georgiev, 544: 128 140, New York Academy of Sciences, New York, 1988

5) Mandala, S. M.; R. A. Thornton, J. Milligan, M. Rosenbach, M. Garcia-Calvo, H. G. Bull, G. Harris, G. K. Abruzzo, A. M. Flattery, C. J. Gill, K. Bartizal, S. Dreikorn \& M. B. Kurtz: Rustmicin, a potent antifungal agent, inhibits sphingolipid synthesis at inositol phosphoceramide synthase. J. Biol. Chem. (submitted)

6) Shirling, E. B. \& D. Gottlieb: Methods for characterization of Streptomyces species. Int. J. Syst. Bacteriol. 16: $313 \sim 340,1966$

7) Hirsch, C. \& J. Sigmund: Use of polymerase chain reaction (PCR) fingerprinting to differentiate bacteria for microbial products screening. J. Ind. Microbiol. 15: $85 \sim 93,1995$

8) Welsh, J. \& M. McClelland: Genomic fingerprints produced by PCR with consensus tRNA gene primers. Nucleic Acids Res. 19: $861 \sim 866,1991$

9) Welsh, J. \& M. MCClelland: PCR-amplified length polymorphisms in tRNA intergenic spacers for categorizing staphylococci. Mol. Microbiol. 6: 1673 1680, 1992

10) Versalovic, J.; T. Koeuth \& J. R. Lupski: Distribution of repetitive DNA sequences in eubacteria and application to fingerprinting of bacterial genomes. Nucl. Acids Res. 19: $6823 \sim 6831,1991$

11) Louws, F. J.; D. W. Fulbright, C. TAylor Stevens \& F. J. DEBruIJN: Specific genomic fingerprints of phytopathogenic Xanthomonas and Pseudomonas pathovars and strains generated with repetitive sequences and PCR. Appl. Environ. Microbiol. 60: 2286 2295, 1994

12) Harris, G. H.; A. Shafiee, M. Angeles Cabello, J. E. Curotto, O. Genilloud, K. E. Goklen, M. B. Kurtz, P. M. Salmon, R. A. Thornton, D. L. Zink \& S. M. MANDALA: Inhibition of fungal sphingolipid biosynthesis by rustmicin, galbonolide $\mathrm{B}$ and their new 21-hydroxy analogs. J. Antibiotics (submitted)

13) Bergey's Manual of Systematic Bacteriology. Volume 4. Ed., S. T. Williams et al., pp. 2418 2450, Williams \& Wilkins, 1989

14) VoBIS, G.: The genus Actinoplanes and related genera. In The Prokaryotes: Second Edition, Volume II. Ed., A. Balows et al., pp. 1029 1060, Springer-Verlag, New York Inc., 1992

15) Wiedmann-Al-Ahmad, M.; H.-V. Tichy \& G. Schon: Characterization of Acinetobacter type strains and isolates obtained from wastewater treatment plants by PCR fingerprinting. Appl. Environ. Microbiol. 60: 4066 4071, 1994 